\title{
Acidosis tubular renal. Presentación de 2 casos clínicos
}

\author{
EDECIA OCHOA*, GILDA BORIE **, MIRIAM CORIES **.
}

La acidosis tubular renal es una entidad nosológica en que hay incapacidad de acidificar la orina por insuficiencia tubular, con función glomerular poco, o nada alterada.

Las acidosis tubulares pueden ser primarias, si la incapacidad de producir orinas ácidas es el único defecto, o secundarias si esta incapacidad se asocia a varias disfunciones renales o sistémicas. Así pues, hay acidosis tubulares secundarias en los sindromes de Fanconi y Lowe, en la intoxicación por vitamina $\mathrm{D}$, en las hipergamaglobulinemias, en el hiperparatiroidismo primario, etc.

Hace varias décadas, Allbright y Butler (1) describieron el sindrome que lleva ese nombre y que se caracteriza por enanismo, raquitismo, litiasis urinaria y nefrocalcinosis, con orinas alcalinas.

Por su parte, Lightwood había llamado la atención sobre una patología especial que se presentaba en niños menores de 18 meses. Estos pacientes tenían falta de desarrollo, vómitos y constipación pertinaz que comenzaba entre el $4{ }^{\circ}$ y $6^{\circ}$ mes de vida; bioquímicamente había una acidosis hiperclorémica con orina neutra o alcalina, y el estudio radióógico mostraba calcificaciones renales en un tercio de los casos.

Posteriormente, el mejor conocimiento de la fisiopatología y el desarrollo de los estudios funcionales renales permitieron diferenciar 2 situaciones que llevan a graves acidosis metabólicas sistémicas por pérdida de bicarbonato en la orina. Se describen entonces, las acidosis tubulares primarias de tipo proximal y de tipo distal. $(3,4$, $5,6,7,8,9,10$ ).

Las acidosis tubulares de tipo proximal (3) se presentan en niños varones de corta edad que

\footnotetext{
- Médico de Servicio de Nefrología, Hospital Roberto del Rio y del Departamento de Medicina Experimental, Sede Norte.

- Químico Farmacéutico, Laboratorio Exploración Renal, Hospital Roberto del Río.
}

clínicamente tienen como única manifestación un retardo de crecimiento. En estos lactantes las orinas son alcalinas, a los niveles plasmáticos de bicarbonato habituales; sin embargo, al hacer descender estos niveles bajo el umbral de excreción se logra acidificar adecuadamente la orina, con eliminación de elevadas cantidades de amonio y acidez titulable.

Se trata pues de un defecto de absorción de bicarbonato en el túbulo proximal, que se traduce en el estudio funcional en umbrales de excreción bajo lo normal. El fluido tubular que escapa del proximal es rico en bicarbonato y la capacidad del distal de acidificar la orina es sobrepasada.

La pérdida continua de bicarbonato lleva a acidosis sistémica y para corregirla se necesitan cantidades muy elevadas de alcalinizantes. El pronóstico es bueno, ya que habitualmente es un cuadro transitorio.

Las acidosis tubulares de tipo distal corresponden a la forma clásica descrita en adultos en que hay una incapacidad de establecer un adecuado gradiente de $\mathrm{pH}$ entre la sangre y el líquido tubular distal, pese a los niveles plasmáticos muy descendidos de bicarbonato.

Los estudios funcionales demuestran que la reabsorción proximal y el umbral de excreción urinaria son normales, pero en el túbulo distal no se excretan hidrogeniones lo que determina baja excreción de acidez titulable y amonio.

Las acidosis tubulares distales se presentan en pacientes generalmente mayores de 2 años, aunque puede encontrarse el antecedente de vómitos, constipación, anorexia, poliuria, deshidratación y crecimiento lento desde los primeros meses de edad. Hay dolores óseos y fracturas patológicas que motivan muchas veces la consulta al médico. También hay debilidad muscular y has- 
ta parálisis periódica derivadas de la constante pérdida de potasio.

En este trabajo se presentan 2 casos de acidosis tubular distal de evolución diferente, analizándose las posibilidades terapéuticas.

Caso $N^{o}$ 1: J. T. P., varón, 4 o hijo nacido de parto normal: Crecimiento normal hasta los 14 meses de edad; desde esa época: pérdida de peso y deformidad en las extremidades inferiores que dificulta la marcha. Comienza a caminar a los 2 años 5 meses, sufriendo caídas que le ocasionaron fractura del fémur derecho. Recibió numerosas dosis de Vit. D $600.000 \mathrm{U}$. Consulta por primera vez en noviembre 1963, a la edad de 4 años 5 meses; pesa $11,100 \mathrm{Kg}$. y mide $82 \mathrm{~cm}$., tiene marcados signos de raquitismo y deformaciones óseas torácicas y de extremidades inferiores, con angulación femoral y patológica, masas musculares hipotróficas con hipotonía e hiporreflexia. Signo de Chvosteck positivo. Se comprueba polidipsia y poliuria de más o menos 2 litros diarios.

El estudio radiológico muestra fracturas patológicas femorales, nefrolitiasis a derecha y exclusión renal izquierda. Edad ósea: 1 año 6 meses.

Los exámenes de laboratorio demuestran acidosis hiperclorémica importante con $\mathrm{pH}$ en sangre: 7.28 y pH urinarios superiores a 6 . Calcemias alrededor de $10 \mathrm{mg} \%$ y calciuria de $15 \mathrm{mg} / \mathrm{Kg} /$ día. Velocidad de filtración glomerular $=52 \mathrm{ml} / \mathrm{min} / 1.73 \mathrm{~m}^{2}$.

Se hace diagnóstico de sindrome de Lightwood Butler Allbright.

Se interviene en mayo 1964 realizándose neo abocamiento ureterovesical bilateral y extrayéndose 3 cálculos. El control post operatorio muestra persistencia de litiasis renal izquierda y nefrocalcinosis derecha.

Los urocultivos post operatorios muestran presencia de Pseudomonas aruginosas resistentes. Se trata con solución citratada, calcio y Vitamina D; corrigiéndose parcialmente la acidosis metabólica.

Estudio de acidificación: Sangre: $\mathrm{pH}=7,19 ; \mathrm{CO}_{2}$ total $=14,5 \mathrm{mM} / 1$. Orina $: \mathrm{pH}=5,97 ; \mathrm{AT}=2,04 \mathrm{uEq} /$ $\mathrm{ml} ; \mathrm{NH}_{4}=5,6 \mathrm{uEq} / \mathrm{ml} ; \mathrm{HCO}_{3}=4,2 \mathrm{uEq} / \mathrm{ml}$ Glucosuria (一).

Es dado de alta controlándose ambulatoriamente en Consultorio de Nefrología.

En 1968, a los 8 años de edad se reevalúa comprobándose una pielonefritis crónica, elevación de la uremia a $124 \mathrm{mg} \%$ con V.F.G. $19 \mathrm{ml} / \min \left(1.73 \mathrm{~m}^{2}\right)$.

Se reemplaza el citrato de sodio por $\mathrm{HCO}_{3}-\mathrm{Na}$ aumentando progresivamente las dosis para compensar la acidosis.

En 1972 tiene 13 años de edad, pesa $19,700 \mathrm{Kg}$ y mide $112 \mathrm{~cm}$; se rehospitaliza por descompensación de la acidosis $(\mathrm{pH}$ sangre $=6.98)$.

Presenta crisis de tetania que no cede con la administración de Ca; calcemia $=7,2 \mathrm{mg} \%$ en los días siguientes tiene crisis convulsivas tónico-clónicas con mordedura de lengua y pérdida de conciencia, se comienza tratamiento con fenobarbital. Las calcemias persisten bajas; al aumentar el aporte de Ca sólo se consi- gue aumento de las calciurias por lo que no se insiste en esta terapia.

Después de una cura con carbencilina (Carpen R) los urocultivos son negativos.

A los 14 años deja de recibir $\mathrm{HCO}_{3}$ - por algunos días por error y debe rehospitalizarse. Se comprueba anemia arregenerativa normocrómica; V.F.G. $=5,4$ $\mathrm{ml} / \mathrm{min} / 1.73 \mathrm{~m}^{2}$ con creatininemias verdaderas de 3.68 $\mathrm{mg} \%$.

Sangre: $\mathrm{pH}$ sangre venosa $=7,29 ; \mathrm{pCO}_{2}=37 \mathrm{~mm}$ $\mathrm{Hg} ; \mathrm{HCO}_{3}$ st $=17,6 \mathrm{mEq} / \mathrm{lt} ; \mathrm{BE}=-7,9$. Orina: $\mathrm{pH}$ 7,$48 ; \mathrm{AT}=(-) ; \mathrm{NH}_{4}=1,5 \mathrm{uEq} / \mathrm{min} / 1,73 \mathrm{~m}^{2}$.

Se repone la terapia con $12 \mathrm{~g}$ de bicarbonato de $\mathrm{Na}$.

Ultimamente ha presentado elevación de la presión arterial que ha hecho modificar el tratamiento.

Se planteó a los familiares la posibilidad de trasplante renal pero no aceptaron.

Diagnóstico: Acidosis tubular renal distal.

Pielonefritis crónica.

Insuficiencia renal global terminal.

Caso No 2: B. A. C., $8^{\text {a }}$ hija, nacida de parto normal. Desarrollo normal hasta los 5 años. A esa edad presenta cambios de conducta, está decaída, apática, baja de peso y se queja de dolores óseos de extremidades inferiores que le impiden la marcha y pérdida de fuerza de los brazos. Tres meses antes del ingreso presenta polidipsia y poliuria.

Días antes del ingreso presenta estado nauseoso y tos por lo que consulta médico, siendo hospitalizada a la edad de 6 años 10 meses (octubre 1973) Examen físico: Peso $14,5 \mathrm{~K}$. Talla $101 \mathrm{~cm}$. Hipotrofia muscular generalizada con disminución de fuerza, respiración acidótica, estertores húmedos en hemitorax derecho. Presión arterial 100/70, deshidratación moderada.

Exámenes de Laboratorio: Sangre: Glicemia $=92$ $\mathrm{mg} \%$; Hematocrito $=44,5 \%$; Uremia $=44,8 \%$; Astrup $\mathrm{pH}$ sangre venosa $=7,217 ; \mathrm{pCO}_{2}=18,7 \mathrm{~mm} \mathrm{Hg}$; $\mathrm{HCO}_{3}-\mathrm{st}=10,6 \mathrm{mEq} / 1 \mathrm{t} ; \mathrm{HCO}_{3}-$ act $=7 \mathrm{mEq} / \mathrm{lt} ;$ $\mathrm{BE}=-19 \mathrm{mEq} / \mathrm{lt} ; \mathrm{BB}=27,1 \mathrm{mEq} / \mathrm{lt}$. Ionograma: $\mathrm{Na}=128,1 \mathrm{mEq} / \mathrm{lt} ; \mathrm{K}=2,85 \mathrm{mEq} / \mathrm{lt} ; \mathrm{Cl}=110$ $\mathrm{mEq} / \mathrm{lt} ; \mathrm{Ca}=7,4 \mathrm{mg} \%$. Orina: Glucosuria (-); Densidad $=1002 ; \mathrm{pH}=6,3 ; \mathrm{Ca}=9 \mathrm{mg} / \mathrm{Kg} /$ día.

Se hace el diagnóstico de acidosis tubular renal de tipo distal.

Radiografís óseas: Acentuada desmineralización, edad ósea: 3 años. No se demostró nefrocalcinosis.

Se trata con bicarbonato de sodio parenteral y luego oral, $\mathrm{K}$ y gluconato de calcio.

Se descartó mala absorción intestinal y el trazado electroforético de proteínas plasmáticas fue normal.

Se controla ambulatoriamente recibiendo a diario: 8 gr de $\mathrm{HCO}_{3}-$ de sodio, 2 gr de gluconato de $\mathrm{Ca}$ oral, $5.000 \mathrm{U}$. de Vit. D y 2 gr de $\mathrm{KCl}$.

Se reevalúa 1 año después. Está asintomática, con buen estado general pero la madre informa que se resiste 


\begin{tabular}{|c|c|c|c|c|c|c|c|c|}
\hline & $\begin{array}{l}\widetilde{\Xi} \\
\text { ఏ } \\
\end{array}$ & $\begin{array}{c}\hat{0} \\
\substack{x \\
+\infty \\
\infty \\
\infty}\end{array}$ & $\begin{array}{l}5 \\
8 \\
0 \\
0 \\
0 \\
0\end{array}$ & 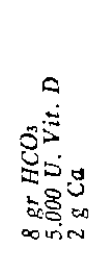 & 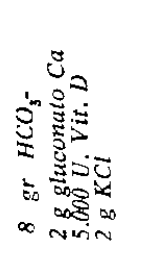 & 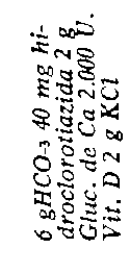 & 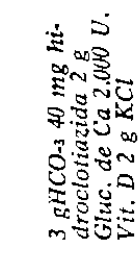 & 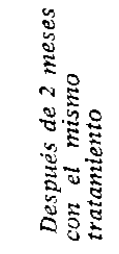 \\
\hline Sangre $\mathrm{pH}$ & 7,217 & 7,41 & 7,36 & & 7,45 & 7,413 & 7,415 & 7,335 \\
\hline $\mathrm{pCO}_{2}(\mathrm{mmHg})$ & 18,7 & 35,5 & 28 & & 42 & 53 & 33,5 & 36 \\
\hline $\mathrm{HCO}_{3}-$ st $(\mathrm{mEq} / \mathrm{lt})$ & 10,6 & 22 & 18 & & 28 & 30 & 23 & 19,5 \\
\hline $\mathrm{HCO}_{3}-$ act $(\mathrm{mEq} / \mathrm{lt})$ & 7 & 25,5 & 14,2 & & 29 & 33 & 21 & 18,5 \\
\hline $\mathrm{BE}(\mathrm{mEq} / \mathrm{lt})$ & -19 & $-1,5$ & $-8,2$ & & +4 & $+7,2$ & $-1,1$ & -6 \\
\hline $\mathrm{Ca}(\mathrm{mg} \%)$ & 7,2 & 6,8 & 8,5 & 9,5 & 10,6 & 8,7 & 10 & 10 \\
\hline$P(\mathrm{mg} \%)$ & $\longrightarrow$ & 2,6 & 3,85 & 4,3 & 5,6 & 4,7 & 5,5 & 4,4 \\
\hline $\mathrm{Na}(\mathrm{mEq} / \mathrm{lt})$ & 128,2 & 140 & 142 & & - & 138 & 136 & \\
\hline $\mathrm{K}(\mathrm{mEq} / \mathrm{lt})$ & 2,85 & 3,1 & 5,4 & & - & 4,0 & 4,2 & \\
\hline $\mathrm{Cl}(\mathrm{mEq} / \mathrm{lt})$ & 110 & 99 & 109 & & - & 90 & 96 & \\
\hline Orina $\mathrm{pH}$ & 6,3 & 8,1 & 7,26 & & 7,64 & 6,97 & 7,26 & \\
\hline $\operatorname{AT}\left(\mathrm{uEq} / \mathrm{min} / 1.73 \mathrm{~m}^{2}\right)$ & 一 & - & 1,7 & & 一 & 3,1 & 1,2 & \\
\hline $\mathrm{NH}_{4}\left(\mathrm{uEq} / \mathrm{min} / 1.73 \mathrm{~m}^{2}\right)$ & 一 & 14,4 & 15,4 & & 8,1 & 3,7 & 10,7 & \\
\hline $\mathrm{HCO}_{3}-\left(\mathrm{uEq} / \mathrm{min} / 1.73 \mathrm{~m}^{2}\right)$ & 一 & 66,1 & 71,7 & & 81,4 & 26,6 & 22,3 & \\
\hline $\mathrm{Ca}(\mathrm{mg} / \mathrm{Kg} /$ día $)$ & 9 & 5 & 6 & 2,2 & 7,5 & 4 & 1,8 & 8,9 \\
\hline $\mathrm{P}(\mathrm{mg} / \mathrm{Kg} /$ día $)$ & - & - & 13 & 27 & 18,5 & 11 & 11,7 & 20 \\
\hline $\begin{array}{l}\text { Dep. de creatinina } \\
\left(\mathrm{ml} / \mathrm{min} / 1.73 \mathrm{~m}^{2}\right)\end{array}$ & 68,6 & 148 & & & 105,8 & 一 & 32 & 117,2 \\
\hline $\begin{array}{l}\text { Osm } \\
\text { Diuresis } 24 \mathrm{hrs} .\end{array}$ & & & & & $\begin{array}{l}215 \text { mos. } \\
2420\end{array}$ & 1196 & 810 & 2550 \\
\hline
\end{tabular}

a tomar el bicarbonato, tiene 7 años 11 meses, pesa $20,300 \mathrm{Kg}$, mide $110 \mathrm{~cm}$, la edad ósea es de 5 años, no hay nefrocalcinosis ni nefrolitiasis. Fosfatasas alcalinas 3.9 U.B. Aminoaciduria normal.

Se trata con hidroclorotiazida $40 \mathrm{mg} /$ día, variando la dosis de bicarbonato de sodio.

Se resumen los exámenes de control en el Cuadro 1.

COMENTARIo. Los casos presentados corresponden al tipo clásico o distal de acidosis tubulares renales.

Cabe destacar que en J. T. la sintomatología se inició muy temprano y la primera consulta fue por graves deformidades óseas por fracturas patológicas. El tratamiento alcalinizante en este niño se hizo en forma irregular, interrumpiéndose en períodos en que dejó de concurrir al Consultorio externo. Un factor agravante de extraordinaria importancia fue la infección urinaria a Pseudomonas que unida a la nefritis intersticial propia de la nefrocalcinosis llevó a la rápida destrucción del parénquima y a la insuficiencia renal crónica global.

Al corregir la acidosis no se logró descenso de la calciuria y el uso transitorio de Vit. D y suplemento de $\mathrm{Ca}$ no modificó la calcemia extrema- damente baja notándose incluso tendencia a aumentar la excreción de calcio urinario; por lo que no se siguió esta terapia.

En B. A.C., en cambio, la sintomatología se inició más tardíamente y en el momento de hacer el diagnóstico no se comprobó nefrocalcinosis radiológica. El tratamiento con alcalinizantes bien controlado consiguió hacer desaparecer la acidosis metabólica disminuyendo notablemente la excreción de $\mathrm{Ca}$ urinario con mejoría ósea y aceleración del crecimiento.

El tratamiento clásico de la acidosis tubular distal es de tipo sintomático ya que se desconoce su etiología $(11,12)$. Se han reportado buenos resultados usando tratamientos alcalinizantes con bicarbonato de sodio y potasio o sus precursores, citratos o lactatos $(13,14,15,16,17,18)$. La poción de Schol que combina citratos de $\mathrm{Na}$ y $K$ con frecuencia es mal tolerada en niños y el tratamiento puede interrumpirse por lo cual es muy recomendable usar bicarbonato de sodio y de potasio si se puede disponer de este último.

El uso de Vit. D y suplementos de $\mathrm{Ca}$ se efectúa después de estabilizar la calciuria al corregir acidosis sistémica. Es necesario el uso de $\mathbf{K}$, cloruro o gluconato que es mejor tolerado. 
Se ha propuesto también el uso de diuréticos, especialmente hidroclorotiazida en los pacientes con A.T.R. (19, 20, 21).

El mecanismo de acción no está bien claro y probablemente esté relacionado con el efecto clorurético y de contracción del espacio extracelular (22) que mejora la absorción proximal de bicarbonato. Además es bien conocido el efecto de la hidroclorotiazida que disminuye la excreción de calcio urinario.

En pacientes con A.T. proximal se han obtenido mejores resultados que en aquéllos con acidosis distales $(20,21)$.

La terapia mixta con hidroclorotiazida y alcalinizantes tendría la ventaja de poder disminuir las dosis de bicarbonatos o citratos, haciendo el tratamiento más cómodo para el paciente.

En nuestra paciente B. A. C. se usó hidroclorotiazida $2 \mathrm{mg} / \mathrm{Kg} /$ día, lográndose mejoría parcial de la acidosis sistémica, disminución de la diuresis y un claro descenso de la V. F. G. El efecto fue transitorio ya que a $\operatorname{los} 2$ meses se reprodujo la acidosis, en ausencia de sintomatología. Aun cuando no se pueden sacar conclusiones, esta observación nos obliga a ser cautos en el uso de hidroclorotiazida y desde luego, a controlar muy estrictamente los índices de laboratorio.

\section{RESUMEN}

Se presentan 2 casos de acidosis tubular renal de tipo distal, a propósito de los cuales se caracteriza la enfermedad y se comentan las posibilidades terapéuticas.

\section{REFERENCIAS}

1.-Allbright F.; Burnett CH Parson, W. Reifenstein $E C$; Roos A. Osteomalacia and late rickets. Medicine 25: 399, 1946.

2.-Lightwood, R; Payne W. W.; Black, J. A. Infantile renal acidosis Pediatrics 12: 628, 1953.

3.-Rodriguez Soriano, J.; Boichis, H.; Stark, H.; Edelmann, C. $M$. Proximal Renal Tubular. Acidosis = a defect in bicarbonate reabsorption. Pediat Res. 1: 81, 1967.

4.-Rodríguez Soriano, J.; Boichis, H.; Edelmann, C. Bicarbonate reabsorption and Hydrogen ion excretion in children with renal tubular acidosis. J. of Pediat. 71: 802, 1967.

5.-Edelmann, C. M.; Boichis, H.; Rodriguez Soriano, J.; Stark, $H$. The renal response of children to acute ammonium chloride acidosis. Pediat Res. 1: 452, 1967.

6.-Broyer, M.; Proesmans, W.; Royer, $P$. La titration des bicarbonates chez l'enfant normal au cours de diverses néphropathies. Rev. Franc. Etud Clin. Biol. 14: $556,1969$.
7.-Morris, Rc. Renal Tubular Acidosis. Mechanism, classification and implications. New Eng. J. Med. 281: $1405,1969$.

8.-Royer, P.; Broyer, M. L'acidose rénale au cours des tubulapathies congénitales. Actualités Néphrologiques de l'Hopital Necker. Editions Médicales Flammarion, Paris, 1967.

9.-Toursel, $F$. Exploration de la founction rénale $\mathrm{d}^{*}$ acidification par les épreuves de surchage acide chez l'enfant. Rev. de Pédiat: 8: 115, 1972.

10.-Rodriguez Soriano, J. The renal regulation of acide-base. Balance and the disturbances noted in renal tubular acidosis. Pediat Clin. of North America 18: 529, 1971.

11.-Strauss, M. B.; Welt, L. G. Enfermedades del riñón. El Ateneo, Buenos Aires, 1966.

12.-Royer, P.; Mathieu, M.; Habib, R. Problemas actuaies de Nefrología infantil. Ediciones Torey. Barcelona, 1965.

13.-Maccioni, A.; Saitua, M. T.; Oxman, S. Mecanismos renales y extrarrenales de compensación en un caso de acidosis tubular crónica idiopática. Rev. Chilena de Pediat. 39: 395, 1968.

14.-Dundon, Sheamus. Treatment of osteomalacia of renal tubular acidosis. Lancet 2: 1204, 1972.

15.-Stickler, G. B.; Bergen, B. J. A review: Short stature in renal disease. Pediat. Res. 7: 978, 1973.

16.-Richards, P.; Chamberlain, M. J.; Wrong, O. $M$. Treatment of osteomalacia of renal tubular acidosis by sodium bicarbonate alone. Lancet 2: 994, 1972.

17. - Lin, S.; Chan, J. C. M. Classical (distal) renal tubular acidosis: endogenous acid production. Pediat. Res. 8: 457, 1974.

18.-Farrell, R. M.; Horwith, M.; Muecke, E. C. Renal tubular acidosis and nephrocalcinosis diagnosis and clinical management. J. of Urol. 11: 429, 1974.

19.-Heidbieder, E.; Heuniemann, H.; Heidland, A.; Krempien, B. K. Treatment of renal tubular acidosis and osteomalacia by salidiuretics. Lancet 1 : 52, 1973.

20._Guigard, J. P.; Torrado, A. Proximal renal tubular acidosis in vitamin D. Deficiency rickets. Acta Pediat. Scand. 62: 543, 1973.

21.-Rampini, S.; Fanconi, A.; Illig, R.; Prader, A. Effect of hydrochlorothiazide on proximal renal tubular acidosis in a patient with idiopathic "de ToniDebré Fanconi syndrome". Helv. Paediat. Acta 23: 13, 1968.

22.-Kurtzman, N.A. Regulation of renal bicarbonate reabsorption by extracellular volumen $\mathbf{J}$. of Clin. Invest. 49: 586, 1970.

Se agradece la cooperación prestada por la Sra. IIse Oberhauser, Sra. Olga Basulto, Srta. Cecilia Silva, Sra. Yolanda Arriagada, del Departamento de Medicina Experimental, Sede Norte. 